\title{
The DLR-Crawler: A Testbed for Actively Compliant Hexapod Walking Based on the Fingers of DLR-Hand II
}

\author{
M. Görner, T. Wimböck, A. Baumann, M. Fuchs, T. Bahls, \\ M. Grebenstein, Ch. Borst, J. Butterfass and G. Hirzinger
}

\begin{abstract}
Walking is a fascinating way of locomotion that is very robust, especially in unstructured terrain. Many researchers devote their time to understanding its underlying principles and to build robots based on their findings. Using the fingers of DLR-Hand II a six-legged actively compliant walking robot is developed. It is intended to be used as testbed for the evaluation of different force- and position-based leg and gait control algorithms for hexapod walking in rough terrain. Following a brief overview of the finger hardware, the use of fingers as legs is analyzed and discussed. The body geometry as well as the systems constituting the robot are described. The compliance control algorithm used is explained and finally some experimental results are presented.
\end{abstract}

\section{INTRODUCTION}

Walking fascinates researchers since decades and much research has been devoted to the understanding of the underlying processes in various animals as well as in humans. Based on the findings, several walking robots have been build that have two [1], four [2], six [3],[4], or even eight [5] legs. The large interest in walking robots lies in their omnidirectional mobility and their ability to traverse unstructured and irregular terrain, which is not feasible for wheeled locomotion. Nevertheless, due to their complexity up to now many walking robots are still outperformed by wheeled or tracked vehicles, but are very promising for the future. The present research on walking robots is mainly driven by two fields of application. In case of two-legged walking, humanoid robots gained importance due to an expected demand for service robots that operate in domestic environments. The second important field of application are search and rescue scenarios as well as terrestrial and extraterrestrial exploration. For these purposes mainly four-, six- and eight-legged robots are of interest. Many of these robots are inspired somehow by nature and borrow in their kinematic structure as well as in parts of their motion pattern generation for example from scorpion [5] or stick insect [3],[6]. Neurobiology suggests that central pattern generators are used to generate basic motion that is adapted to the current situation by use of sensor information and overlayed by reflex responses in case of unforeseen events. Beside motion generation another difficult and interfering problem is leg coordination. For the stick insect Cruse [7] found very effective coordination principles that are partially used in the control of six-legged robots like Tarry from the University of Duisburg [6] or the walking robot from the Technical University Munich [3]. So far, there are still many

DLR - German Aerospace Center, Institute of Robotics and Mechatronics, Oberpfaffenhofen, Germany, ( martin.goerner@dlr.de ) mechanisms in walking, which are not well understood and are currently under investigation. One problem for example is how to incorporate force and torque information in the generation of motion and leg coordination.

The DLR-Crawler described in this paper is a walking hexapod robot based on the fingers of the DLR-Hand II. It is designed as an experimental testbed for leg and gait control algorithms that offers joint torque and foot forcetorque sensing in addition to the commonly used position sensors. To the authors, there is currently no other hexapod robot known offering joint torque control based on joint torque sensing. Thus, it enables the use of compliance and impedance control algorithms and gives the possibility to incorporate force and torque information in the generation of walking patterns.

The paper is organized as follows. Section II discusses the use of fingers as legs. In section III the crawler design process is described and the final system is presented. Section IV deals with joint compliance control and a first tripod gait for system tests. Finally, section V shows results of the performance in first experiments.

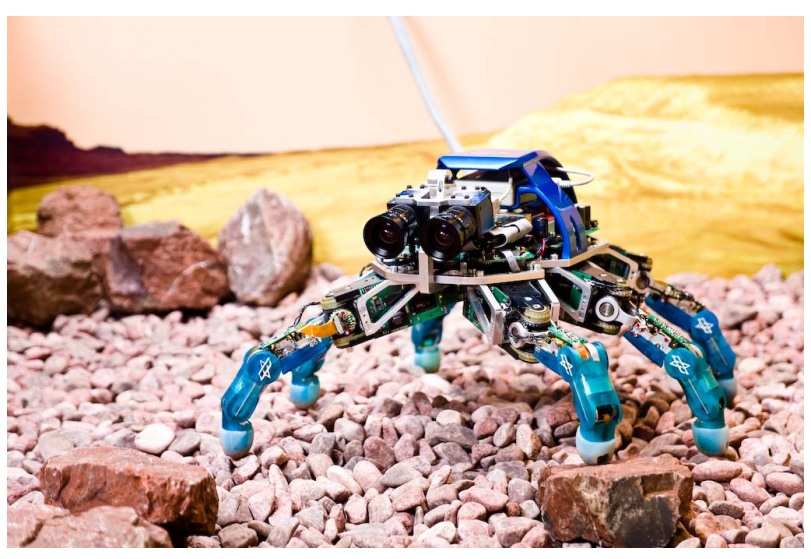

Fig. 1. DLR-Crawler

\section{ARE THE DLR-HAND II FINGERS SUITED TO BE USED AS LEGS}

The development of highly integrated legs is not a trivial task. It poses many challenging problems to be solved, ranging from finding a suitable kinematic structure to the choice of actuation system and sensor equipment. It is strongly affected by body geometry, desired mobility and 
payload capabilities as well as the intended terrain to walk on. Since nature evolved very functional legs many robotics scientists draw inspiration from kinematic structures found in a variety of animals. As already mentioned above, for sixlegged walking robots the stick insect is a very prominent example, which inspired the design of the walking robot of the Technical University Munich [3], the Lauron robot of the Research Center for Information Technology in Karlsruhe [4] as well as the Tarry robots built at the University Duisburg [6]. Apart from biologically inspired legs, there also exist robots with leg designs that are technically motivated. One example are the Titan robots from Tokyo University [8], which employ pantograph mechanisms in order to decouple balancing of gravitational loads from producing forward motion.

Since so far no walking robot has been built at our institute, a first prototype using mainly existing components was desired in order to gain experience in the field of walking. Due to their modular design the fingers of the DLRHand II seemed to be suited very well for this purpose. To verify this assumption results of a workspace analysis are presented right after a brief overview of the finger design. At the end of this section the suitability of the fingers to be used as legs is discussed and limiting factors for a crawler design are presented.

\section{A. Finger Mechanics, Sensors, Actuators and Communicati- on System}

Each finger of the DLR-Hand II has four joints and three degrees of freedom since the medial and distal joints are coupled in a ratio of one to one. Figure 2 shows the kinematic structure of the finger. Two degrees of freedom are realized by the proximal joint using a differential bevel gear mechanism, which provides the possibility of additively combining the torques of the two motors that drive the joint to generate a larger torque about one of its axes. Thus, in order to achieve a specified maximum joint torque, motors could be chosen smaller and lighter.



Fig. 2. Kinematic Structure of a finger of DLR-Hand II

The finger joints are actuated by permanent magnet synchronous motors each combined with a harmonic drive and a tooth belt transmissions stage. Apart from the two motors in the finger base, a third motor situated in the proximal link is driving the coupled medial and distal joints. All
TABLE I

TECHNICAL DATA OF A DLR-HAND II FINGER

\begin{tabular}{|l||c|}
\hline \multicolumn{1}{|l||}{ Link Lengths } & \\
\hline proximal link & $75 \mathrm{~mm}$ \\
\hline medial link & $40 \mathrm{~mm}$ \\
\hline distal link & $40 \mathrm{~mm}$ \\
\hline Joint Motion Ranges & $\pm 37 \mathrm{deg}$ \\
\hline abduction/adduction proximal joint & $-55 /+75 \mathrm{deg}$ \\
\hline extension/flexion proximal joint & $-20 /+105 \mathrm{deg}$ \\
\hline extension/flexion medial and distal joint & \\
\hline Transmission Ratios & $100: 1$ \\
\hline harmonic drives & $1.2: 1$ \\
\hline tooth belt proximal joint & $2: 1$ \\
\hline tooth belt medial joint & $>360 \mathrm{deg} / \mathrm{s}$ \\
\hline Maximum Joint Velocity & $375 \mathrm{~g}$ \\
\hline Total Finger Mass & \\
\hline
\end{tabular}

motors are chosen according to the requirement to deliver a force of $30 \mathrm{~N}$ at the fingertip in an outstretched finger configuration. Table I displays some relevant technical data of the finger. In order to achieve good manipulation capabilities each finger is equipped with a variety of sensors. Each joint has a strain gauge based joint torque sensor and a conductive plastic potentiometer for providing an absolute joint position reference. Due to the differential bevel gear design of the proximal joint, the potentiometers are aligned with the driving bevel gears instead of the joint axes directly. Additionally, each motor is equipped with linear hall effect sensors, which deliver a high resolution relative motor position that is used for control and motor commutation. The fingertip includes a miniaturized strain gauge based 6 DOF force-torque sensor to measure manipulation forces and torques. Further, temperature sensors are implemented to allow temperature induced drift compensation of sensor readings as well as to protect the motors. Since most of the computation to control the fingers is performed on an external realtime computer, the DLR- Hand II incorporates a fast hierarchical serial communication system, that collects and transmits data. It is implemented in FPGAs according to the IEEE 1355 standard, which specifies a slim protocol layer, prevents collisions, and supports different physical transmission mediums. Due to the fast communication a 1 $\mathrm{kHz}$ control loop can be closed for the whole hand. More detailed descriptions can be found in [9], [10] and [11].

\section{B. Finger Workspace Analysis}

In order to evaluate whether the finger is suited to be used as leg and to identify appropriate configurations, the workspace is analyzed with respect to a useful motion range, manipulability and force capabilities. Useful motion range means in this case the area that the fingertip can reach in a horizontal plane without any other part of the finger touching this plane. Figure 3 shows the workspace of the finger corresponding to the joint angle ranges given in table I. The figure already suggests to use a horizontal or a slightly against the horizontal tilted finger base to obtain a maximum useful motion range of the fingertip. This motion range is shown by the blue contour for an exemplary vertical distance 
of $\mathrm{z}=50 \mathrm{~mm}$ to the finger base coordinate system, which is situated at the intersection of the axes of the proximal joint.
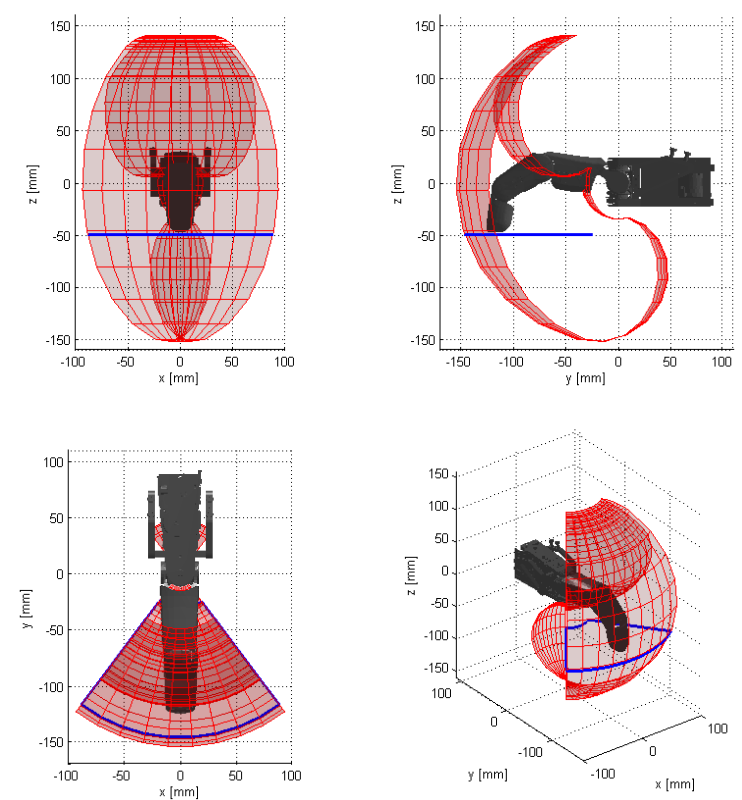

Fig. 3. Workspace of a finger of DLR-Hand II

Figure 4 shows the effect of tilting the finger base by an angle $\alpha$ against the horizontal plane on the afore mentioned useful motion range of the fingertip. It can be seen that for example tilting the base about 30 degree leads to a $60 \%$ increase of the useful motion range. This is very important since the workspace of the finger is small compared to the workspace of the legs, most existing walking robots have.
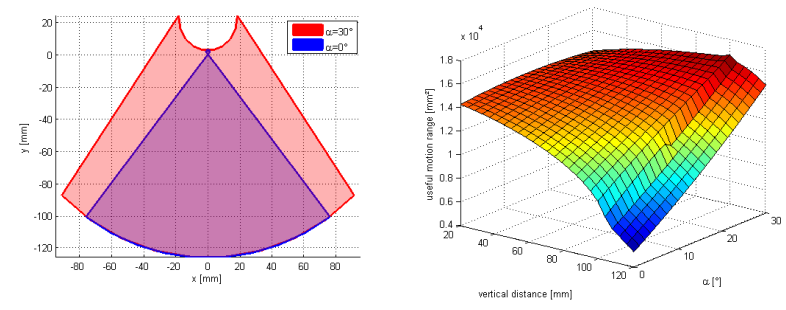

Fig. 4. Comparison useful motion range: left: useful motion range for $\alpha$ $=0 \mathrm{deg}$ and $\alpha=30 \mathrm{deg}$ at $90 \mathrm{~mm}$ vertical distance; right: useful motion range with respect to vertical distance and finger base angle $\alpha$

Beside the useful motion range of the fingertip it is of interest to analyze the transmission of joint velocities to cartesian velocities at the fingertip for different finger configurations. To be suitable as leg it should be possible to move the finger equally well in every direction starting at a configuration, where the fingertip is located at a central position of the workspace. For this purpose a manipulability analysis as described by Yoshikawa [12] is conducted. The Jacobian matrix $\mathbf{J}\left(\boldsymbol{\theta}_{j}\right)=\frac{\partial \mathbf{x}_{t i p}\left(\boldsymbol{\theta}_{j}\right)}{\partial \boldsymbol{\theta}_{j}}$ mapping joint velocities $\dot{\boldsymbol{\theta}}_{j} \in \mathbb{R}^{3}$ onto Cartesian velocities $\dot{\mathbf{x}}_{t i p} \in \mathbb{R}^{3}$ at the fingertip is used.

$$
\dot{\mathbf{x}}_{t i p}=\mathbf{J}\left(\boldsymbol{\theta}_{j}\right) \dot{\boldsymbol{\theta}}_{j}
$$

A unit sphere in joint velocity space is mapped by $\mathbf{J}\left(\boldsymbol{\theta}_{j}\right)$ onto an ellipsoid in Cartesian fingertip velocity space. The lengths of the principle axes of this ellipsoid are constituted by the singular values $\sigma_{1}\left(\boldsymbol{\theta}_{j}\right), \sigma_{2}\left(\boldsymbol{\theta}_{j}\right)$ and $\sigma_{3}\left(\boldsymbol{\theta}_{j}\right)$ of the Jacobian $\mathbf{J}\left(\boldsymbol{\theta}_{j}\right)$. The axis corresponding to the largest singular value is the best direction to generate velocity. Further, the volume of the velocity ellipsoid is a measure for the velocity generation capacity. Using the singular values the volume of the ellipsoid can be calculated by following equation.

$$
V\left(\boldsymbol{\theta}_{j}\right)=\frac{4}{3} \pi \sigma_{1}\left(\boldsymbol{\theta}_{j}\right) \sigma_{2}\left(\boldsymbol{\theta}_{j}\right) \sigma_{3}\left(\boldsymbol{\theta}_{j}\right)
$$

Apart from the factor $\frac{4}{3} \pi$ this is the same as Yoshikawas manipulability measure $w$.

$$
w\left(\boldsymbol{\theta}_{j}\right)=\sqrt{\operatorname{det}\left(\mathbf{J}\left(\boldsymbol{\theta}_{j}\right) \mathbf{J}^{T}\left(\boldsymbol{\theta}_{j}\right)\right)}=\sigma_{1}\left(\boldsymbol{\theta}_{j}\right) \sigma_{2}\left(\boldsymbol{\theta}_{j}\right) \sigma_{3}\left(\boldsymbol{\theta}_{j}\right)
$$

Figure 5 shows the velocity ellipsoid for various finger configurations and figure 6 displays the volume of the velocity ellipsoid with respect to the joint angles. The diagrams at the top of figure 6 show that there is no influence of the angle of joint one on the velocity ellipsoid volume. The diagram at the bottom of this figure shows that the ellipsoid volume is largest for values of $\theta_{2}$ in between -55 degree to 20 degree and values of $\theta_{3}$ in between 20 degree to 60 degree. Thus, in this region the finger has the largest velocity generation capacity. Again by tilting the finger base against the horizontal plane walking could benefit by using a larger part of this region. Another effect in this case is that the vertical fingertip motion comes closely to the axis of the velocity ellipsoid related to the smallest singular value whereas the axes corresponding to the larger singular values almost fall into the horizontal plane. This means the better directions to generate Cartesian velocity at the fingertip lie within the potential walking plane.
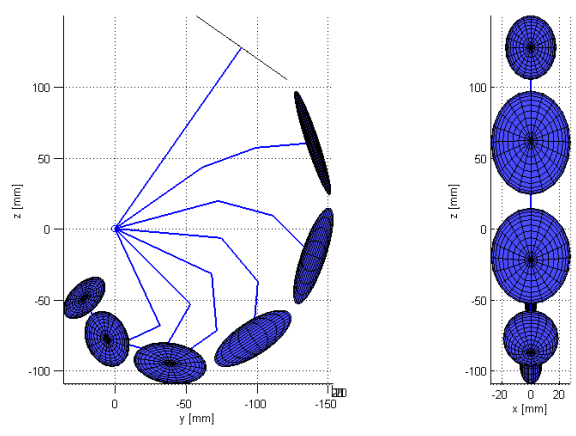

Fig. 5. Velocity ellipsoid for various finger configurations with horizontal base: left: yz-plane; right: xz-plane 

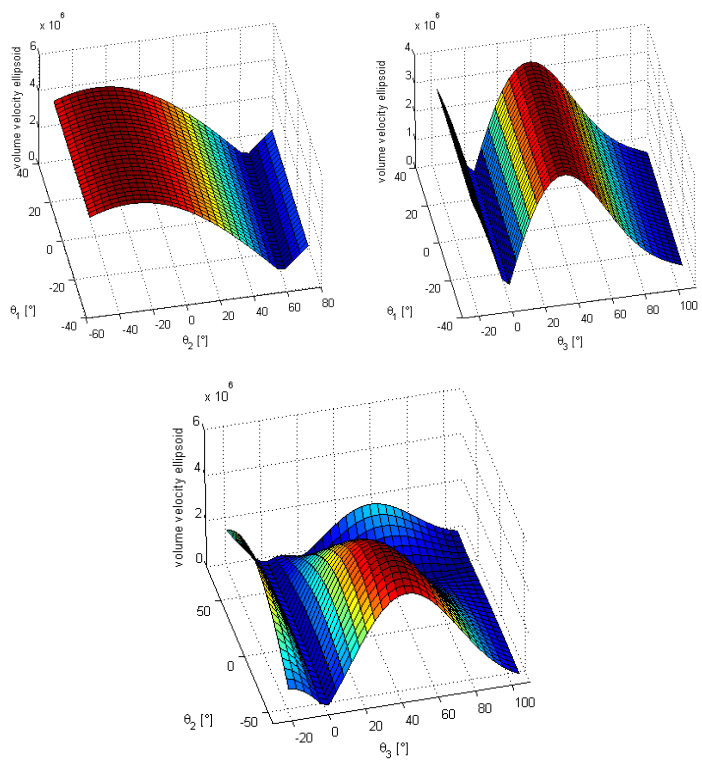

Fig. 6. Volume of the velocity ellipsoid with respect to the joint angles: top left: $\theta_{3}=40 \mathrm{deg}$; top right: $\theta_{2}=10 \mathrm{deg}$; bottom: $\theta_{1}=0 \mathrm{deg}$

Using the static mapping between external forces at the fingertip $\mathbf{f}_{t i p} \in \mathbb{R}^{3}$ and joint torques $\boldsymbol{\tau}_{j} \in \mathbb{R}^{3}$, as given by the following equation, a force ellipsoid can be derived.

$$
\mathbf{f}_{t i p}=\left(\mathbf{J}^{T}\left(\boldsymbol{\theta}_{j}\right)\right)^{-1} \boldsymbol{\tau}_{j}
$$

The inverse of the Jacobian transpose $\left(\mathbf{J}^{T}\left(\boldsymbol{\theta}_{j}\right)\right)^{-1}$ exists for all $\boldsymbol{\theta}_{j}$ except for $\theta_{j, 3}=0$, which is a boundary singularity. The force ellipsoid is dual to the velocity ellipsoid, what means the best direction to generate force coincides with the worst direction to generate velocity. In case of a tilted finger base this direction falls perpendicular to the walking plane and thus aligns with the gravity vector. Hence, the gravitational loads of a walking robot are supported with least effort in this direction.

\section{Discussion: Fingers as Legs}

The above analysis gave useful insight to asses the suitability of the fingers of the DLR-Hand II to be used as legs. The available workspace shows the largest volume below the horizontal plane that includes the base of the finger. This volume allows strokes up to $10 \mathrm{~cm}$ and walking heights in a range of $2 \mathrm{~cm}$ to $12 \mathrm{~cm}$. Depending on the gait, walking speeds up to $20 \mathrm{~cm} / \mathrm{s}$ are possible without reaching the maximum joint velocities. The velocity generation capacity of the finger is largest for values of $\theta_{2}$ ranging from 55 degree to 20 degree and values of $\theta_{3}$ ranging from 20 degree to 60 degree. Tilting the finger base in between 10 degree to 30 degree against the horizontal better uses this region, increases the possible horizontal motion range of the fingertip and decreases the effort to support the robot weight. The walking motion should be mainly generated by the stronger base motors and therefore by abduction and adduction of the finger. The analysis shows that the fingers are suited to be used as legs of a small walking robot. The availability of force, torque and position sensor allows to test different leg controllers as well as different walking pattern generation methods incorporating position, contact force and joint torque information. Nevertheless, increased effort has to be put into the leg coordination due to the limited workspace of the fingers.

\section{CRAWLER DESIGN}

The design process of a walking robot needs decisions about the number of legs to use, the body geometry as well as the general system architecture including sensors, electronics and communication structure. The next section explains the choice of the final body geometry and presents the complete system architecture.

\section{A. Choosing a Geometry}

Finding a good body geometry is a difficult problem due to conflicting requirements for mobility and stability as well as possible terrains to master and changing operation scenarios. For this reason many walking robot designs draw their inspiration from nature. Nevertheless, most robots are built with identical legs, which is not natural. Therefore, for the design technical considerations have to be taken into account.

Considering the results of the above analysis the best configuration for using the fingers as legs is close to the one found in crabs or insects, which have legs pointing away from the sides of their body. Since the fingers possess no passive energy storages that could absorb larger impacts, the use of dynamic gaits is not feasible. Thus, a static gait as the tripod gait or the wave gait found in hexapods is desired. So, a six-legged configuration seems to be most appropriate. An eight-legged robot would also be an option, but to the expense of additional hardware and a more complex control. As already mentioned, requirements for mobility and stability have a strong impact on the robot design. To make the robot more mobile the base joints of all legs should be very close to each other, what at the same time decreases the support polygon ${ }^{1}$ and thus stability. Increasing stability by placing the base joints further apart reduces mobility. Hence, a trade-off has to be found. Using six identical fingers calls for a rotationally symmetric body that has the legs equally distributed around its circumference. With abduction and adduction of the finger as the preferred motion to propel the robot, its body should have an axis representing a preferred motion direction. Due to having identical legs it is sensible to use this axis as an axis of symmetry. Further, the middle pair of legs is placed on this axis dividing it into two equal parts. Since the finger base contains two motors it is a little bulky. Thus, the base joints of the middle legs are placed as close to each other as possible. This fixes the body width at the middle of the robot to $220 \mathrm{~mm}$. All legs are tilted by an angle $\alpha$ of 15 degree against the horizontal plane. This

\footnotetext{
${ }^{1}$ Is a polygon spanned by the parallel projection of foot to ground contact points into a plane orthogonal to the gravity vector.
} 
is a trade-off combining the advantages of a tilted base as discussed in the previous section with a reasonable stepping height. The length of the body $l_{b}$, the distance between the front legs as well as the rear legs $w_{b 2}$ and the angle $\gamma$ shown in figure 7 are subject to an optimization minimizing the vertical contact forces of all legs, while maximizing the distance of the geometrical center of the robot to the boundary of the support polygon over a cycle of a nominal tripod gait in forward direction. This is done in order to find a configuration showing good stability, while bringing the maximum load to an equal level for all legs. The final parameters found for the robot body are $l_{b}=250 \mathrm{~mm}, w_{b 2}$ $=176 \mathrm{~mm}$ and $\gamma=40$ degree.

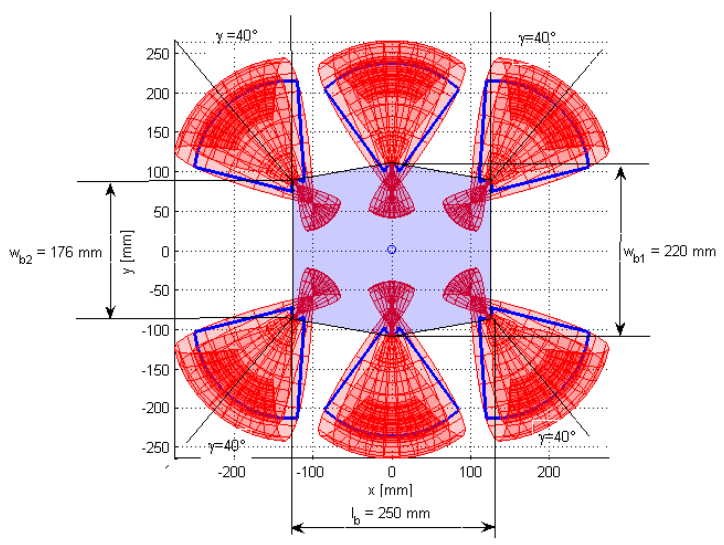

Fig. 7. Body parameters and leg workspaces

\section{B. The System Architecture}

In advance of designing the complete system a decision about the power supply as well as the computational hardware was crucial. Due the fact that the crawler is intended to be a laboratory testbed to evaluate leg and gait control algorithms an external 24 VDC power supply as well as an external QNX based realtime PC to perform all computations were chosen. Due to the cables the motion range of the crawler is currently limited to $10 \mathrm{~m}$.

The body of the crawler consist of a slim aluminum frame, that holds all electronics, the legs and further parts.

For walking and navigation the crawler needs sensors additional to the ones integrated in the DLR-Hand II fingers. The first is a stereo camera head for visual odometry, obstacle avoidance and navigation. Of the camera head two different versions exist, one with low cost cameras and a pan-tilt unit that transmits the camera frames via an analog PALsignal to an external receiver as well as a high resolution firewire stereo camera head that has no actuated pan-tilt unit. Further, an SPI-interface is prepared to equip the crawler with an inertial measurement unit consisting of gyroscopes and acceleration sensors. Additional interfaces are reserved for sensors extensions such as infrared or ultrasound distance sensors.

In order to operate the crawler some new electronics had to be designed. One part is a power converter board that generates all voltages to supply the motors as well as all digital and analog electronics. The electronics are galvanically decoupled from the external 24 VDC supply. Another new part is an interface board that distributes supply voltages and signal lines to all parts of the robot. This board performs signal transformations and galvanically decouples signal lines via optocouplers. An FPGA board based on a Xilinx Virtex-II Pro that was previously developed at the institute is the communication node of the crawler. It is connected via a fiber optic cable to the external PC and implements a router that controls the data exchange between PC, legs and the additional sensors. Figure 8 shows a schematic to clarify this hardware setup and figure 9 shows the assembled robot.

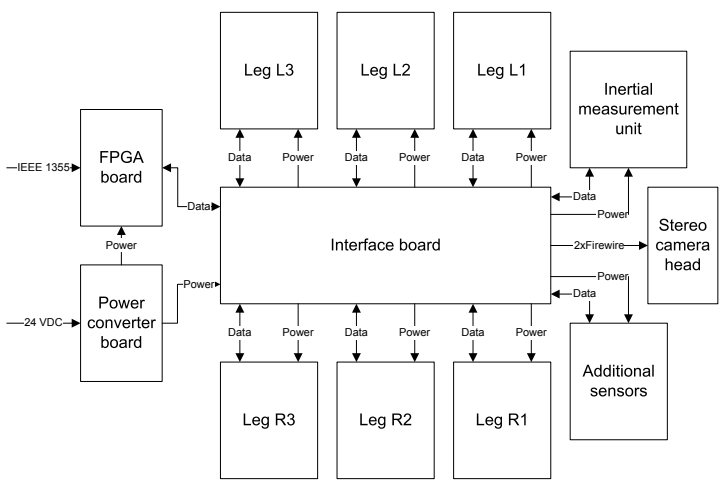

Fig. 8. Crawler hardware concept

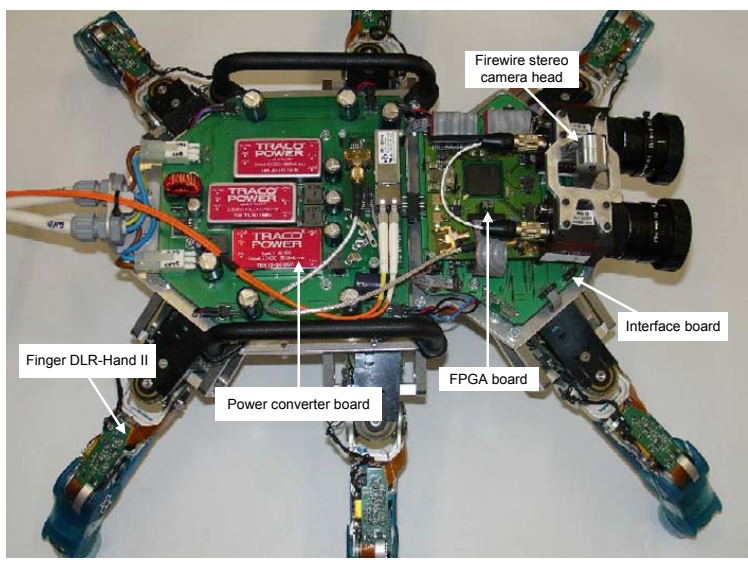

Fig. 9. Assembled DLR-Crawler hardware

Including all parts the robot has a final mass of $3.5 \mathrm{~kg}$ and the capacity to carry a payload equal to its mass.

\section{CRAWLER CONTROL}

Being equipped with joint position sensors, joint torque sensors and a force-torque sensor at the tip of each leg the crawler is an ideal testbed for force and position control 
algorithms as well as a combination of both. A detailed analysis of the influence of contact forces on the generation of walking patterns is possible and different hypotheses might be tested in future. Using controllers developed for the DLR-Hand II Cartesian or joint impedance control can be implemented currently. Compared to pure position control schemes, as applied in many walking robots, internal forces caused by foot placement errors or errors in the kinematic model can be reduced very effectively, leading to a reduction of slipping. Ground contact forces can be controlled and sudden contacts are recognized early. Next the joint compliance control scheme also used for the DLR-Hand II and the generation of a tripod gait as initial gait are presented.

\section{A. Compliance Control}

The joint compliance of a leg can be understood as attaching virtual springs and dampers to each joint and to initiate motion by shifting their virtual equilibrium $\boldsymbol{\theta}_{j, d} \in$ $\mathbb{R}^{3}$. It is implemented based on the following well known compliance control law.

$$
\begin{gathered}
\boldsymbol{\tau}_{j, d}=-\frac{\partial V\left(\boldsymbol{\theta}_{j}\right)}{\partial \boldsymbol{\theta}_{j}}-\mathbf{D}\left(\boldsymbol{\theta}_{j}\right) \dot{\boldsymbol{\theta}}_{j}+\mathbf{g}\left(\boldsymbol{\theta}_{j}\right) \\
V=\left(\boldsymbol{\theta}_{j, d}-\boldsymbol{\theta}_{j}\right)^{T} \mathbf{K}_{\theta}\left(\boldsymbol{\theta}_{j, d}-\boldsymbol{\theta}_{j}\right)
\end{gathered}
$$

Herein, $\boldsymbol{\tau}_{j, d} \in \mathbb{R}^{3}$ is a vector of desired joint torques, $\boldsymbol{\theta}_{j} \in \mathbb{R}^{3}$ a vector of actual joint positions, $\mathbf{g}\left(\boldsymbol{\theta}_{j}\right)$ a vector of gravitational torques and $\mathbf{D}\left(\boldsymbol{\theta}_{j}\right)$ a positive semi-definite damping matrix. $V\left(\boldsymbol{\theta}_{j}\right)$ is a potential function and $\mathbf{K}_{\theta}$ a stiffness matrix that in case of joint compliance control is chosen to be diagonal. The desired joint torques are transformed to motor coordinates, which are fed to an underlying motor torque control loop that consists of a high gain proportional motor torque controller and a disturbance compensation term $\hat{\boldsymbol{\tau}}_{m, \text { fric }}$ as given by equation 7 . This term compensates static and viscous friction as well as the motor back-emf based on the output of a friction observer. The whole control loop is displayed in figure 10.

$$
\mathbf{u}_{m}=\mathbf{K}_{\tau}\left(\boldsymbol{\tau}_{m, d}-\boldsymbol{\tau}_{m}\right)+\hat{\boldsymbol{\tau}}_{m, \text { fric }}
$$

\section{B. First Gait}

For system tests a tripod gait is the first gait implemented. This gait emerges in nature in fast walking insects and is well known. It is characterized by two sets of three legs, that interchange in stance and swing motion. On the crawler it is implemented to be variable in walking speed, step length and height as well as to smoothly transition between forward and backward walking and left and right turning. In order to achieve smooth transitions between swing and stance the underlying pattern is designed by use of a sample trajectory shown in figure 11. The swing phase of the leg motion is generated using fourth order polynomials. To generate the walking pattern this sample trajectory is rotated into the corresponding leg coordinate system and shifted in phase by 50 percent of the step cycle length for one set of legs. The

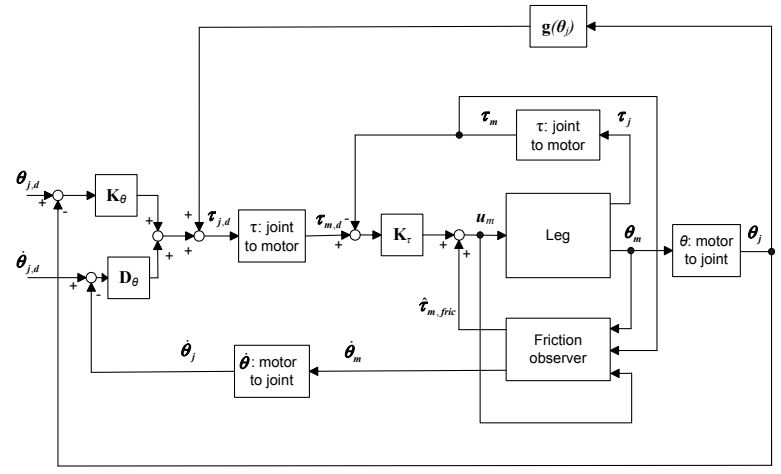

Fig. 10. Joint compliance control scheme for one leg

joint angles are then calculated by inverse kinematics and serve as desired values of the joint compliance controller.
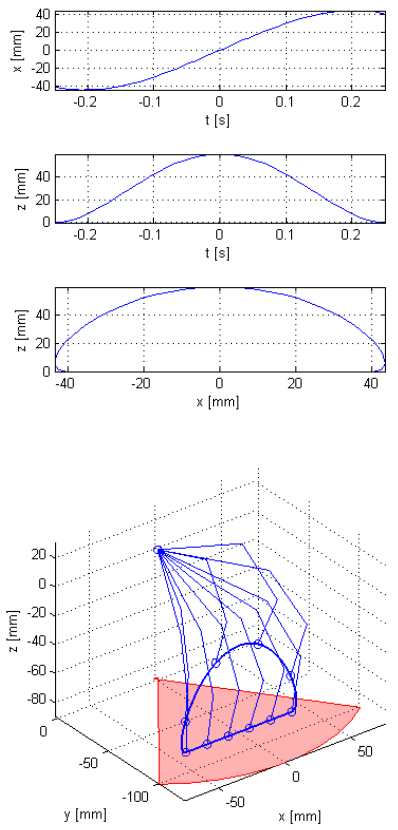

Fig. 11. Sample step cycle: top: Swing phase; bottom: Complete step cycle in relation to the useful motion range of a leg

\section{EXPERIMENTAL RESULTS}

So far, several experiments were conducted to test the crawler hardware and to evaluate the performance of the joint compliance controller. The crawler walked smoothly on flat ground as well as on gravel for a broad range of joint stiffness settings. It reached velocities up to $20 \mathrm{~cm} / \mathrm{s}$ and carried payloads of $3 \mathrm{~kg}$ without performance degradation. Especially for walking on gravel with a gait designed for flat terrain, the benefits of the compliance control became 
apparent. The following diagrams show measurements during walking on carpet. Figure 12 shows the tracking of the desired joint angles for the left hind leg. Tracking errors appear due to the fact that the virtual compliance has to generate the torques to propel the robot and to support its weight. During stance the second and third joint of each leg show the largest deviations of the desired values since they have to support most of the robot weight. In figure 13 the corresponding measured joint torques can be seen.
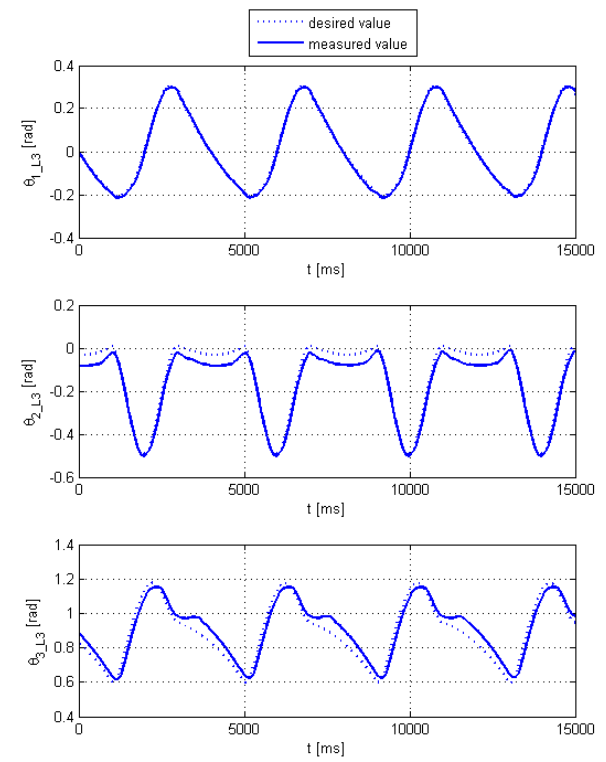

Fig. 12. Joint angles of the left hind leg during tripod gait

\section{CONCLUSIONS AND FUTURE WORK}

\section{A. Conclusions}

The development of a six-legged walking robot based on the fingers of DLR-Hand II was presented. The robot is equipped with joint torque sensors as well as force-torque sensors at the feet enabling compliance and impedance control. First, the suitability of fingers as legs was discussed. Then a body geometry was derived and the system architecture was presented. Finally, results on first tests using a tripod gait were given. They show that the joint compliance control works very well and that the robot walks very smooth. These results are very promising for future gait implementations.

\section{B. Future Work}

In future free gaits based on position and force control will be implemented in order to allow walking in highly unstructured terrain. Visual odometry will be fused with joint angle readings and inertial measurements for robot navigation. Further, obstacle avoidance based on vision will be investigated.

\section{ACKNOWLEDGMENTS}

The authors are very thankful to Mathias Nickl and Stefan Joerg for helping to implement the crawler communication.
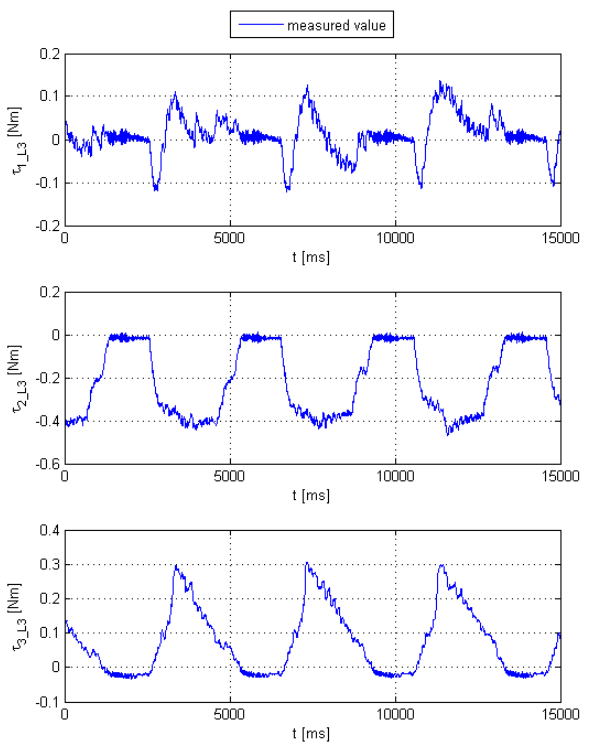

Fig. 13. Joint torques of the left hind leg during tripod gait

\section{REFERENCES}

[1] K. Kaneko, F. Kanehiro, S. Kajita, H. Hirukawa, T. Kawasaki, M. Hirata, K. Akachi and T. Isozumi, Humanoid Robot HRP-2, Proceedings of the 2004 IEEE Conference on Robotics \& Automation, New Orleans, USA , 2004, pp 1083-1090

[2] K. Berns, W. Ilg, M.Deck, J. Albiez and R. Dillmann, Mechanical Constructionand Computer Architecture of the Four-Legged Walking Machine BISAM, IEEE/ASME Transactions on Mechatronics, 1999, Vol. 4, No. 1, pp 32-38

[3] F. Pfeiffer, H.J. Weidemann and J. Eltze, Leg Design Based on Biological Principles, Proceedings of the 1993 IEEE Conference on Robotics \& Automation, Atlanta, USA , 1993, pp 352-358

[4] B. Gassmann, K.U. Scholl and K. Berns, Locomotion of Lauron III in Rough Terrain, International Conference on Advanced Mechatronics, Como, Italy, 2001

[5] D. Spenneberg and F. Kirchner, SCORPION: A biomimetic Walking Robot, Robotik 2002, VDI-Bericht, 2002, Vol. 1679, pp 677-682

[6] M. Frik, M. Guddat, M. Karatas and D. Losch, A Novel Approach to Autonomous Control of Walking Machines, Proceedings of the 2nd International Conference on Climbing and Walking Robots, Portsmouth, $U K, 1999$, pp 333-342

[7] H. Cruse, What Mechanisms Coordinate Leg Movement in Walking Arthropods, Trends in Neuroscience, 1990, Vol. 13, pp 15-21

[8] S. Hirose, K. Yoneda, K. Arai and T. Ibe, Design of Prismatic Quadruped Walking Vehicle TITAN VI, 5th International Conference on Advanced Robotics, 1991, pp 723-728

[9] J. Butterfass, M. Grebenstein, H. Liu and G. Hirzinger, DLR-Hand II: Next Generation of a Dextrous Robot Hand, Proceedings of the 2001 IEEE Conference on Robotics \& Automation, Seoul, Korea, 2001, pp 109-114

[10] S. Haidacher, J. Butterfass, M. Fischer, M. Grebenstein, K. Joehl, K. Kunze, M. Nickl, N. Seitz and G.Hirzinger, DLR-Hand II: Hard- and Software Architecture for Information Processing, Proceedings of the 2003 IEEE Conference on Robotics \& Automation, Taipei, Taiwan, 2003, pp 684-689

[11] Ch. Borst, M. Fischer, S. Haidacher, H. Liu and G. Hirzinger, DLRHand II: Experiments and Experiences with an Anthropomorphic Hand, Proceedings of the 2003 IEEE Conference on Robotics \& Automation, Taipei, Taiwan, 2003, pp 702-707

[12] T.Yoshikawa, Manipulability of Robotic Mechanisms, The International Journal of Robotics Research, 1985, Vol. 4, No.3 